\title{
PROX1 IMMUNOHISTOCHEMICAL ANALYSIS OF PEDIATRIC VASCULAR ANOMALIES, A POSIBLE PROGNOSTIC AND THERAPEUTIC FACTOR OF HEMANGIOMAS?
}

\author{
Rodica Heredea $^{1,2}$, Simona Cerbu ${ }^{3}$, Emil Radu Iacob $^{4}$, Teodora Hoinoiu ${ }^{5}$, \\ Maria-Corina Stănciulescu ${ }^{4}$
}

\section{Abstract}

Infantile and congenital hemangiomas still a challenge for both the pediatric surgeon and the pediatrician. The treatment of hemangiomas must be individualized according to: the type of lesion, location, size, depth, stage of growth and evolution of the lesion. To date, none of the available therapies is considered standard therapy. Currently, personalized therapy is not widely applied in Romania, not being included in the usual therapy protocols in any of the types of hemangioma. This is a starting point for the identification of new specific therapeutic targets that preserve normal endothelial cells and determine the regression of hemangioma, especially recurrent ones and those with an increased proliferation rate. Another controversial and unexplained aspect, consequently unexploited from a therapeutic point of view is represented by the expression and role of lymphatic markers in infantile and congenital hemangiomas.

Keywords: vascular anomalies, PROX-1, hemangiomas, arteriovenous malformations, PDGF B

\section{Introduction}

It is well known that childhood hemangioma is the best known childhood tumor (5-10\% incidence), consisting of endothelial cell proliferation and pericytes [1]. Despite the benign morphopathological character, infantile hemangiomas can have a "malignant" behavior, especially if they appear in organs located at the level of the cranial box or those in the thoracic box. The evolution of hemangiomas is unpredictable, most regress spontaneously, only about $10 \%$ are destructive, disfiguring and endanger the patient's life; the factors that determine the progression, regression and heterogeneity of the response to conventional therapy are not known in totality [2]. All this is due to the lack of a customized molecular profile for each type of hemangioma [3].

Infantile hemangioma can be routinely diagnosed by clinical means and rarely require therapy, but rare vascular tumors are frequently difficult to diagnose, for the diagnosis and treatment of these lesions is important multidisciplinary approach, thorough clinical examination, appropriate imaging and histopathological diagnosis [4]. Thus, the ISSVA classification was developed in 2014 [5] which was revised in 2018, it is based on the relationship between clinical, radiological, histological and molecular aspects of vascular anomalies [6].

Recently, the histopathological classification of hemangiomas has been revised, but recurrences following discontinuation of adjuvant or combination drug therapy could not be explained.

The expression of lymphatic markers is inconsistent and there is currently no clear classification and therapeutic orientation based on their existence. It seems that the expression of lymphatic markers is variable, depending on the topography of hemangiomas, but also on the evolutionary stage $[7,8,9]$.

Platelet-derived growth factor (PDGF) is little studied in infantile vascular malformations. The same is true for Prox 1 or CLIC1. PDGF is reported to be expressed in hemangiomas in only 22 of articles in PubMed while Prox 1 is associated with hemangiomas with spindle type morphology [10]. Prox 1 (prospero homeobox 1 protein) is an endothelial transcription factor considered a regulator of lymphatic endothelial differentiation, it is expressed in the nuclei of the developing and adult lymphatic endothelial cells $[11,12,13,14,15]$. Prox 1 is also expressed in other epithelial tissues, more frequently liver and pancreas, especially during development $[16,17,18]$.

${ }^{1}$ Department of Pathology, "Louis Turcanu" Children's Clinical Emergency Hospital, Victor Babes University of Medicine and Pharmacy, Timisoara, Romania,

${ }^{2} \mathrm{PhD}$ Student, Victor Babes University of Medicine and Pharmacy, Timisoara, Romania,

${ }^{3}$ Department of Radiology, Victor Babes University of Medicine and Pharmacy, Timisoara, Romania,

${ }^{4}$ Department of Pediatric Surgery, Victor Babes University of Medicine and Pharmacy, Timisoara, Romania

${ }^{5}$ Department of Clinical Practical Skills, Victor Babeş University of Medicine and Pharmacy, Timisoara, Romania

E-mail: heredea.rodica@yahoo.com,cerbusimona@yahoo.com,radueiacob@umft.ro,tstoichitoiu@umft.ro,

stanciulescu.maria@umft.ro 
The studied cases are divided, according to the ISSVA 2018 classification, into vascular tumors- 41 cases and simple vascular malformations - 9 cases (table 1). Vascular tumors are infantile and congenital hemangiomas (33 cases), glomangiomas (2 cases), pyogenic granulomas (5 cases) and one of Kaposiform hemangioendothelioma.

Table 1. - Classificasion of vascular anomalies from the study.
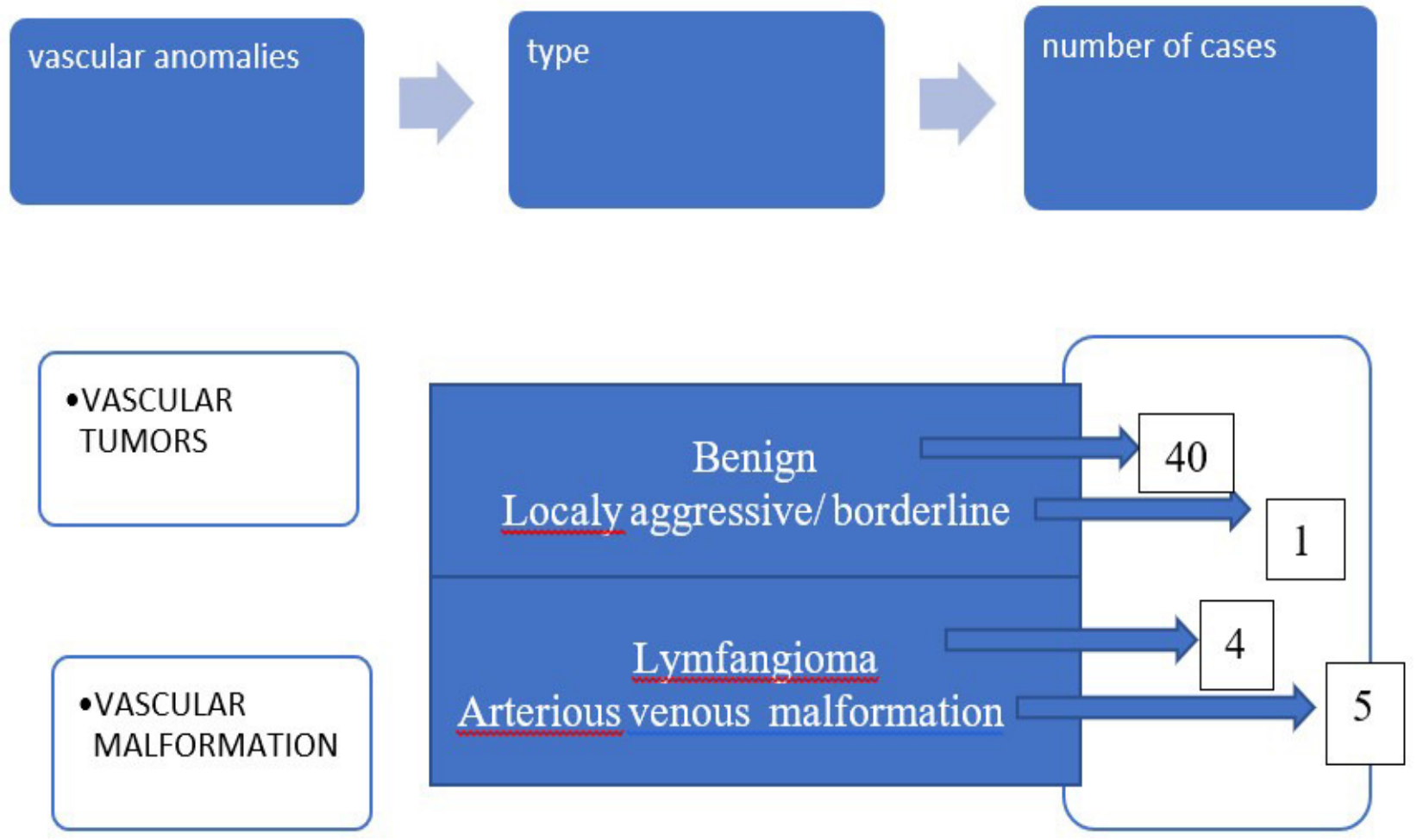

\section{-VASCULAR MALFORMATION}

\section{Methods}

The tissue fragments taken were within the size limits standard, having less than $1 \mathrm{~cm}$.

Sampling was followed by fixation of the fragments. This step was performed in 10\% buffered formalin for 48 hours. This was followed by the removal of excess fixative by washing with running water for two hours. Following the removal of excess fixative, the parts were embedded in paraffin.

The sectioning was performed with the microtome. The $3 \mu$ sections thus obtained were spread on silane slides and then spread hot, in medium aqueous. The drying process followed, performed at a temperature of $37^{\circ} \mathrm{C}$. The slides were thus exposed for 20-30 minutes.

Deparaffining was performed over a period of 30 minutes in a benzene bath at $57{ }^{\circ} \mathrm{C}$. Two baths of benzene followed, lasting 10 minutes each, at room temperature. For rehydration, the dewaxed sections were passed for 10 minutes in individual alcohol baths, concentrations of $100 \%$, $96 \%, 80 \%, 70 \%$. For another 10 minutes, each alcohol bath is followed by a bath with distilled water. At the end of all these stages, the sections are ready for coloring.

The cases selected for the immunohistochemical technique were stained by simple reactions using the following primary antibodies Prox 1 and PDGF B.

\section{Results}

Of the 50 cases studied are divided, according to the ISSVA 2018 classification, into vascular tumors (41 cases) and simple vascular malformations ( 9 cases), from the figure below it can be seen that most cases are represented by vascular tumors (figure 1).

Most cases of infantile and congenital hemangioma were identified in females (25 cases), this distribution correlates with data from the literature [19]. Lymphangioma and muscular hemangioma were found in the female population, and pyogenic granuloma in boys. The figure 2 shows the sex distribution of hemangiomas and arteriovenous malformations. 


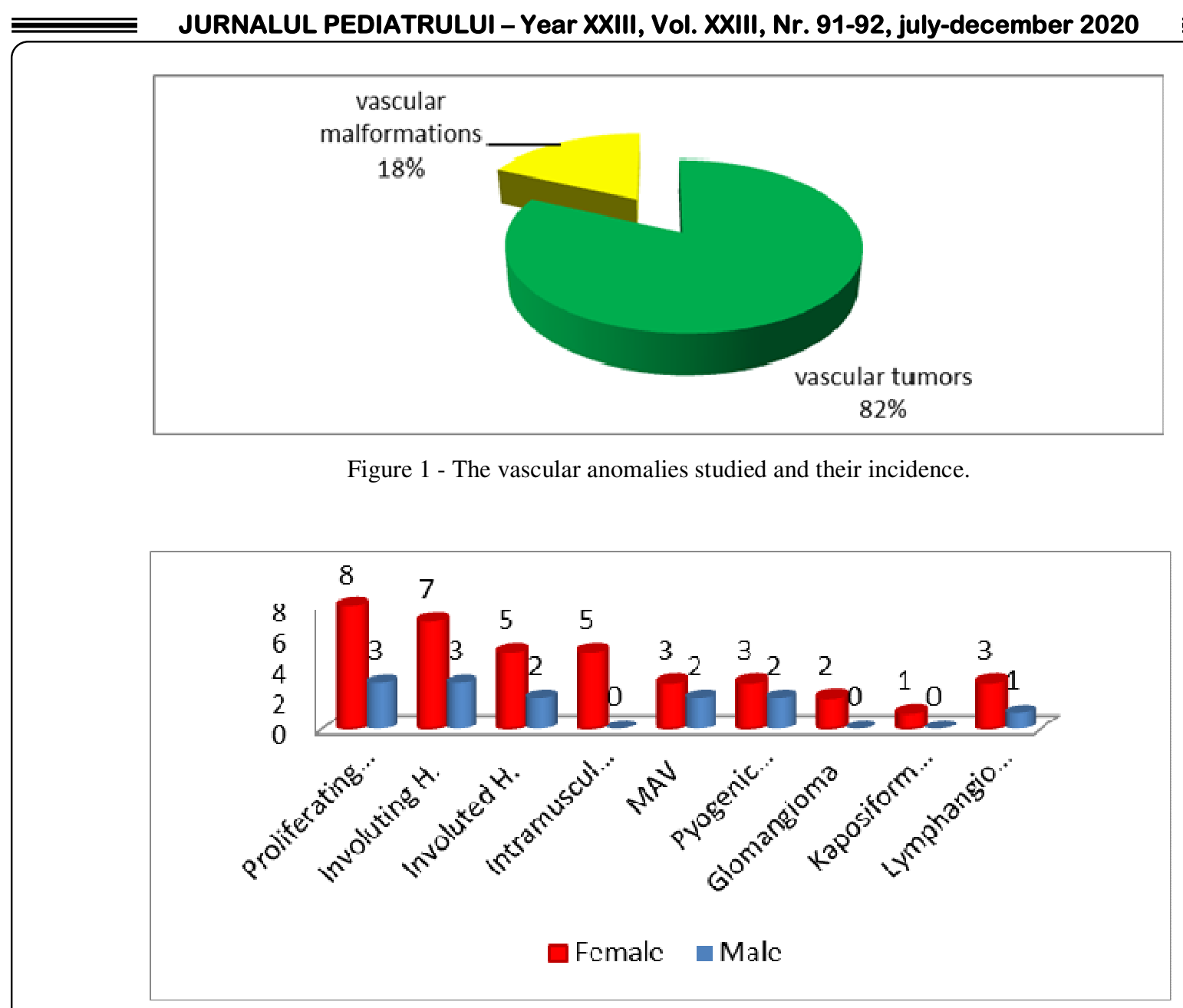

Figure 2 - Vascular anomalies, sex distribution.

The cases of infantile and congenital hemangiomas included in the study were classified according to the predominance of solid areas of endothelial cells and the density of vascular structures with lumens evident in: proliferating, involuting and involuted hemangioma

Hemangiomas in the proliferating and involuting phase were diagnosed in children aged between 2 months and two years, most of them were highlighted in the first year of life. Changes corresponding to an involuted phase of hemangioma appear from the first year of life and were found until the age of 12 years. The youngest patient who was diagnosed with intramuscular hemangioma was 4 years old and the oldest 17 years old. Arterio-venous malformation has been identified in children older than 2 years.

In the hemangioma the lobes are composed of capillarytype vascular structures, packed, some with a well-visible lumen or endothelial cells arranged in the form of beaches / islands. In the proliferative phase (fig. 3a), solid masses and capillaries packed with inconstantly evident lumens predominate. Hemangioma in the involuting phase (fig. 3b) has thicker septa, the lobules contain more vascular structures and less solids, they are directly proportional to the type of involution. The vascular structures are lined with swollen endothelial cells, some of which have a hobnail appearance. In the hemangioma involuted in the lobules richer fibrous and adipose tissue is identified, the vessels have a larger caliber, a wall of variable thickness and isolated in the lumen, thrombi can be identified in different evolutionary stages. On Giemsa staining, a higher number of mast cells was identified in proliferative and involuting hemangiomas and fewer in involuted hemangiomas.

Intramuscular hemangioma (fig. 3c) present striated muscle fibers dissected by a proliferation of vascular structures with variable lumen, most with cavernous appearance, focal with interanastomotic appearance, similar to arterial-venous malformation. The wall is thin, lined with cubic endothelial cells, swollen, without atypia. Some vascular structures show thrombi at various stages of evolution, isolated with aspects of intravascular papillary endothelial hyperplasia [20]. The stroma presents with an inflammatory lymph-plasma cell infiltrate, foamy histiomacrophages, giant multinucleated cells, hemosiderin deposits and isolated dystrophic calcifications. 


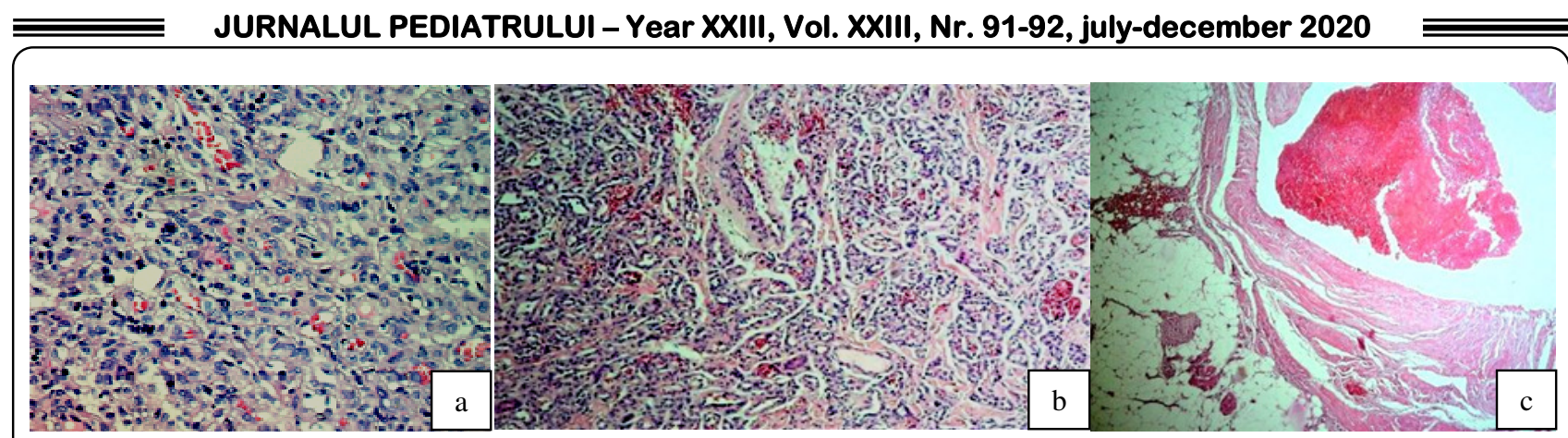

Figure 3 - a) proliferating hemangioma, b) involuting hemangioma, c) intramuscular hemangioma.

The figure 4 shows the correlation of the lesions with the anatomical location. It can be seen that vascular tumors are most commonly located in the trunk and region of the head or neck, while arterio-venous malformations are common in the limbs. Proliferative hemangioma was identified in the trunk, head and neck, and was not found in the limbs.
Depending on the histopathological characters and the intensity of PROX1 expression, infantile hemangiomas were divided into four categories: proliferative, involuting early, involuting tardive and involuted (table 2).

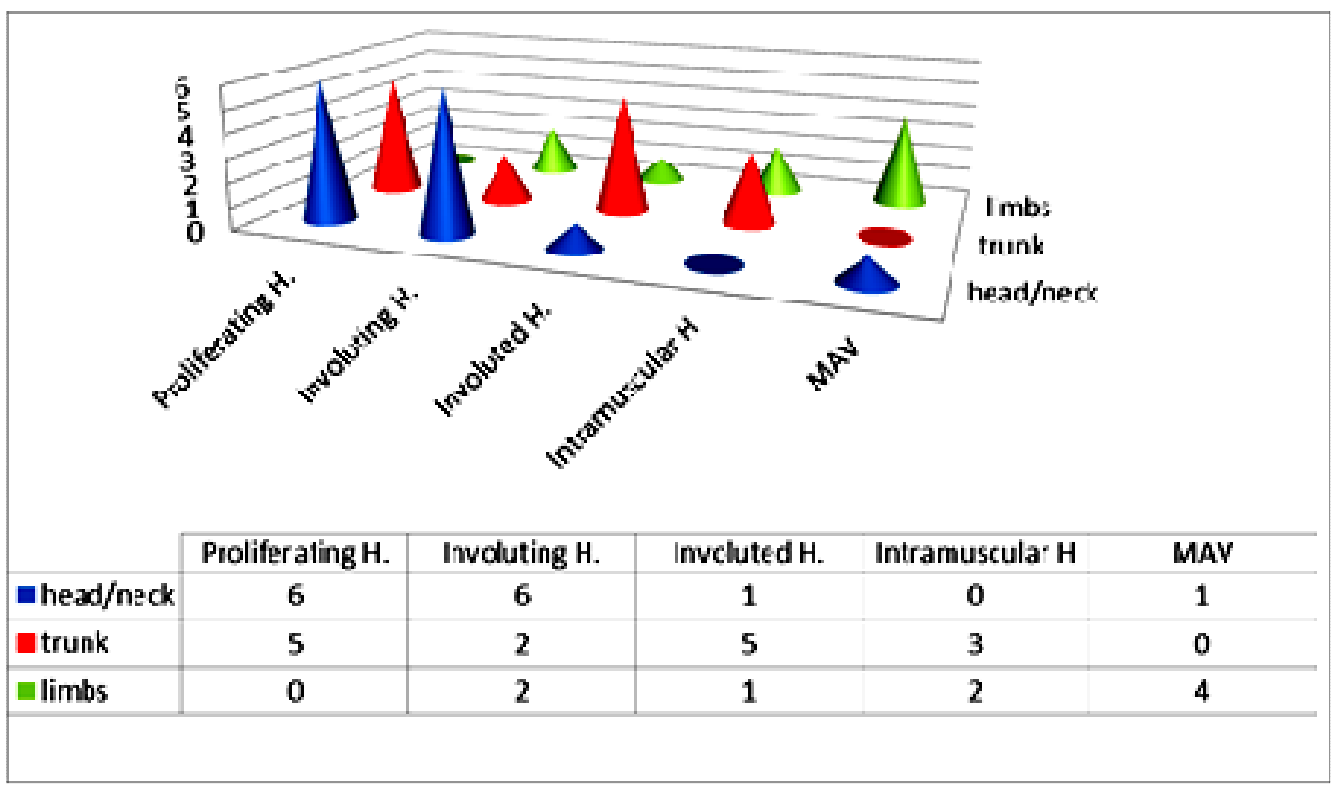

Figure 4 - Anatomical distribution of hemangiomas and arterio-venous malformations.

Table 2 - Vascular anomalies and PROX1 reaction intensity.

\begin{tabular}{|l|l|}
\hline LESION & PROX1 \\
\hline Intramuscular hemangioma & 3 \\
\hline Infantile hemangioma proliferative phase & 0 \\
\hline Infantile hemangioma involuting phase-early & 1 \\
\hline Infantile hemangioma involuting phase-tardive & 2 \\
\hline Infantile hemangioma involuted phase & 3 \\
\hline Kaposiform hemangioenothelioma & 0 \\
\hline Pyogenic granuloma & $2 / 3$ \\
\hline Glomangioma & 0 \\
\hline Lymfangioma & $0 / 3$ \\
\hline Arterio venous malformation & $0 / 1 / 3$ \\
\hline
\end{tabular}




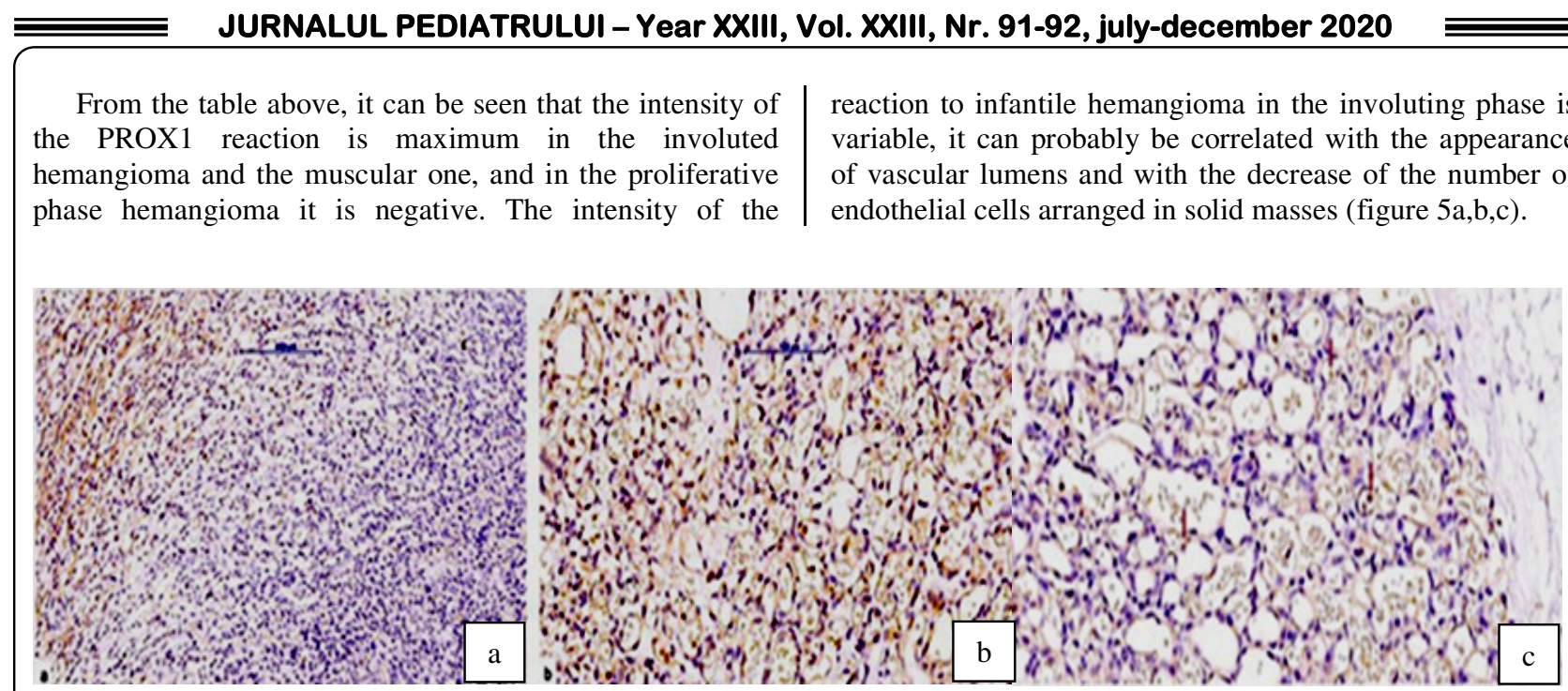

Figure 5 - Prox 1 expression in undifferentiated areas (a), differentiated (b). Note the lack of Prox 1 in undifferentiated areas and the gradual appearance in differentiating $(a, b)$ and differentiated $(c)$ areas.

PDGF $B$ expression is variable in infantile hemangiomas, so in the proliferative phase most have the intensity of reaction 1 ( 5 cases) and only one case has the intensity 3 , probably a late proliferation phase. The intensity of the reaction is variable as the hemangioma evolves, most of those involuted have a maximum intensity of the reaction. A maximum intensity of the PDGF $B$ reaction is also identified in the case of arterio-venous malformations.

In the cases studied there were two cases of treated hemangiomas. In the one treated with propranolol the intensity of the PROX reaction is 3 , it was considered an involuted hemangioma. The second case is a proliferative hemangioma treated with propranolol (without results, it grew and ulcerated), which was surgically excised, cauterized and then relapsed, the PROX1 reaction is negative.

Intramuscular hemangiomas were all diagnosed in female patients, in the upper or lower limbs and, as a particular aspect, all cases showed a +3 reaction to Prox 1 .

Involutive hemangiomas were analyzed using the same criteria as for proliferative hemangiomas.

The global analysis of involutive hemangiomas demonstrated a statistically significant correlation between PDGF-BB and PROX1 expression (Table 3 and Figure 6).

\begin{tabular}{|c|c|c|c|}
\hline \multicolumn{4}{|c|}{ Correlation Matrix } \\
\hline & & PDGF-BB & PROX1 \\
\hline \multirow[t]{8}{*}{ PDGF-BB } & Pearson's r & - & $0.738 *$ \\
\hline & $p$-value & - & 0.001 \\
\hline & $95 \%$ Cl Upper & - & 0.903 \\
\hline & 95\% CI Lower & - & 0.382 \\
\hline & Spearman's tho & - & $0.753 \cdots$ \\
\hline & p-value & - & $<.001$ \\
\hline & Kendall's Tau B & - & $0.696 *$ \\
\hline & $p$-value & - & 0.004 \\
\hline \multirow[t]{8}{*}{ PROX 1} & Pearson's r & $0.738^{*}$ & - \\
\hline & p-value & 0.001 & - \\
\hline & $95 \%$ Cl Upper & 0.903 & - \\
\hline & 95\% CI Lower & 0.382 & - \\
\hline & Spearman's tho & $0.753 \cdots$ & - \\
\hline & p-value & $<.001$ & - \\
\hline & Kendall's Tau B & $0.696 *$ & - \\
\hline & p-value & 0.004 & - \\
\hline
\end{tabular}

Note. $" p<.05, " p<.01, \cdots p<.001$ riot

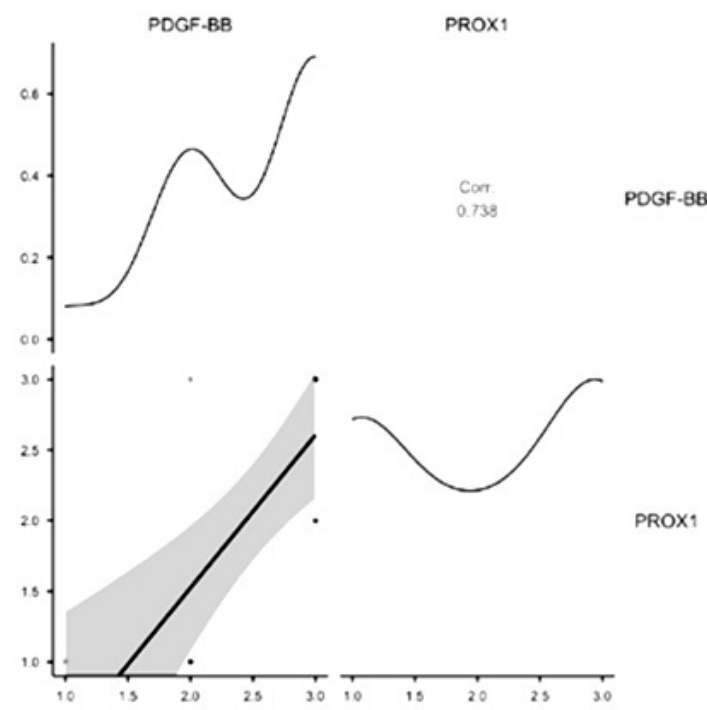

Table 3 / Figure 6 - Correlation between PROX1 and PDGF-BB in involutive hemangiomas. 


\section{Discussions}

The highest number of cases of proliferating and involuting hemangiomas were excised from the head and neck region, and the involuted hemangioma is located predominantly on the trunk. Arterio-venous malformations were most frequently excised from the limbs, having the same location as the data in the literature [21].

In the studied cases, the hemangioma is frequently located in the dermis with extension in the subcutaneous cellular tissue and consists of lobules separated by fibrous septa containing vascular structures of larger caliber, in variable numbers [22]

In this study we immunohistochemically examinated the expression of Prox 1 protein, who is known to be expressed in lymphangiomas and lymphatic endothelium $[11,13,14,15]$, but Prox 1 is involved in development of heart, liver, and pancreas. [16,18,23]. In our study intensity of the PROX1 reaction is maximum in the involuted hemangioma and the muscular one, and in the proliferative phase hemangioma it is negative.

Intramuscular hemangiomas were all diagnosed in the upper or lower limbs and, as a particular aspect, all cases showed a +3 reaction to Prox 1 .

It remains to be determined whether Prox 1 contributes to clinico-pathologic and evolution distinction of hemangioma vs. vascular malformation [24].
Further studies are needed to determine whether there is a correlation of Prox1 with other lymphatic / endothelial markers or with clinio-pathological aspects [25].

\section{Conclusions}

In our study, the benign lesions were the majority, and among them most were infantile and congenital hemangiomas.

Proliferative infantile hemangioma has been located on the trunk, head and neck.

Vascular tumors are most commonly located on the head,neck, trunk region, while vascular malformations are frequently found in the limbs.

Arteriovenous malformations were most commonly located on the limbs, having the same location as the data in the literature.

The intensity of the PROX1 reaction is maximum in the involved hemangioma and the muscular one. In the proliferative phase of hemangioma it is negative.

The intensity of the reaction to involute phase of hemangioma is variable, it can probably be correlated with the appearance of vascular lumens and with the decrease the number of endothelial cells arranged in solid masses.

Our study demonstrates the need to establish multidisciplinary teams that will aim to standardize methods of diagnosis and treatment of vascular anomalies.

\section{References}

1. Mulliken JB, Enjolras O: Congenital hemangiomas and infantile hemangioma: Missing links. J Am Acad Dermatol 50:875-882, 2004

2. Della Rosa N, Bertozzi N, Adani R. Vascular malformation and their unpredictable evolution: A true challenge for physicians. Acta Biomed. 2020 Aug 25;91(3):e2020067. doi: 10.23750/abm.v91i3.8298. PMID: 32921762; PMCID: PMC7716989.

3. Ricci KW. Advances in the Medical Management of Vascular Anomalies. Semin Intervent Radiol 2017; 34 : 239-249

4. Wildgruber M, Sadick M, Müller-Wille R, Wohlgemuth WA. Vascular tumors in infants and adolescents. Insights Imaging. 2019 Mar 13;10(1):30. doi: 10.1186/s13244-019-0718-6. PMID: 30868300 ; PMCID: PMC6419671.

5. Wassef M, Blei F, Adams D. et al. Vascular Anomalies Classification: Recommendations From the International Society for the Study of Vascular Anomalies. Pediatrics 2015; 136: e203-e214

6. Sadick M, Müller-Wille R, Wildgruber M, Wohlgemuth WA. Vascular Anomalies (Part I): Classification and Diagnostics of Vascular Anomalies. Rofo. 2018 Sep;190(9):825-835. English. doi: 10.1055/a-06208925. Epub 2018 Jun 6. PMID: 29874693.

7. Nonogaki S, Campos HG, Butugan O, Soares FA, Mangone FR, Torloni H, Brentani MM. Markers of vascular differentiation, proliferation and tissue remodeling in juvenile nasopharyngeal angiofibromas. Exp Ther Med. 2010 Nov;1(6):921-926.

8. Le Huu AR, Jokinen CH, Rubin BP, Mihm MC, Weiss SW, North PE, Dadras SS. Expression of prox1, lymphatic endothelial nuclear transcription factor, in Kaposiform hemangioendothelioma and tufted angioma. Am J Surg Pathol. 2010 Nov;34(11):1563-73.

9. Benoit MM, North PE, McKenna MJ, Mihm MC, Johnson MM, Cunningham MJ. Facial nerve hemangiomas: vascular tumors or malformations? Otolaryngol Head Neck Surg. 2010 Jan;142(1):108-14.

10. Wang L, Gao T, Wang G. Expression of Prox1, D2-40, and WT1 in spindle cell hemangioma. J Cutan Pathol. 2014;41(5):447-450. doi:10.1111/cup.12309.

11. Hirakawa S, Hong YK, Harvey N, Schacht V, Matsuda K, Libermann T, Detmar M. Identification of vascular lineage-specific genes by transcriptional profiling of isolated blood vascular and lymphatic endothelial cells. Am J Pathol. 2003 Feb; 162(2):575-86.

12. Johnson NC, Dillard ME, Baluk P, McDonald DM, Harvey NL, Frase SL, Oliver G. Lymphatic endothelial cell identity is reversible and its maintenance requires Prox1 activity. Genes Dev. 2008 Dec 1;22(23):3282-91. doi: 10.1101/gad.1727208. PMID: 19056883; PMCID: PMC2600759. 


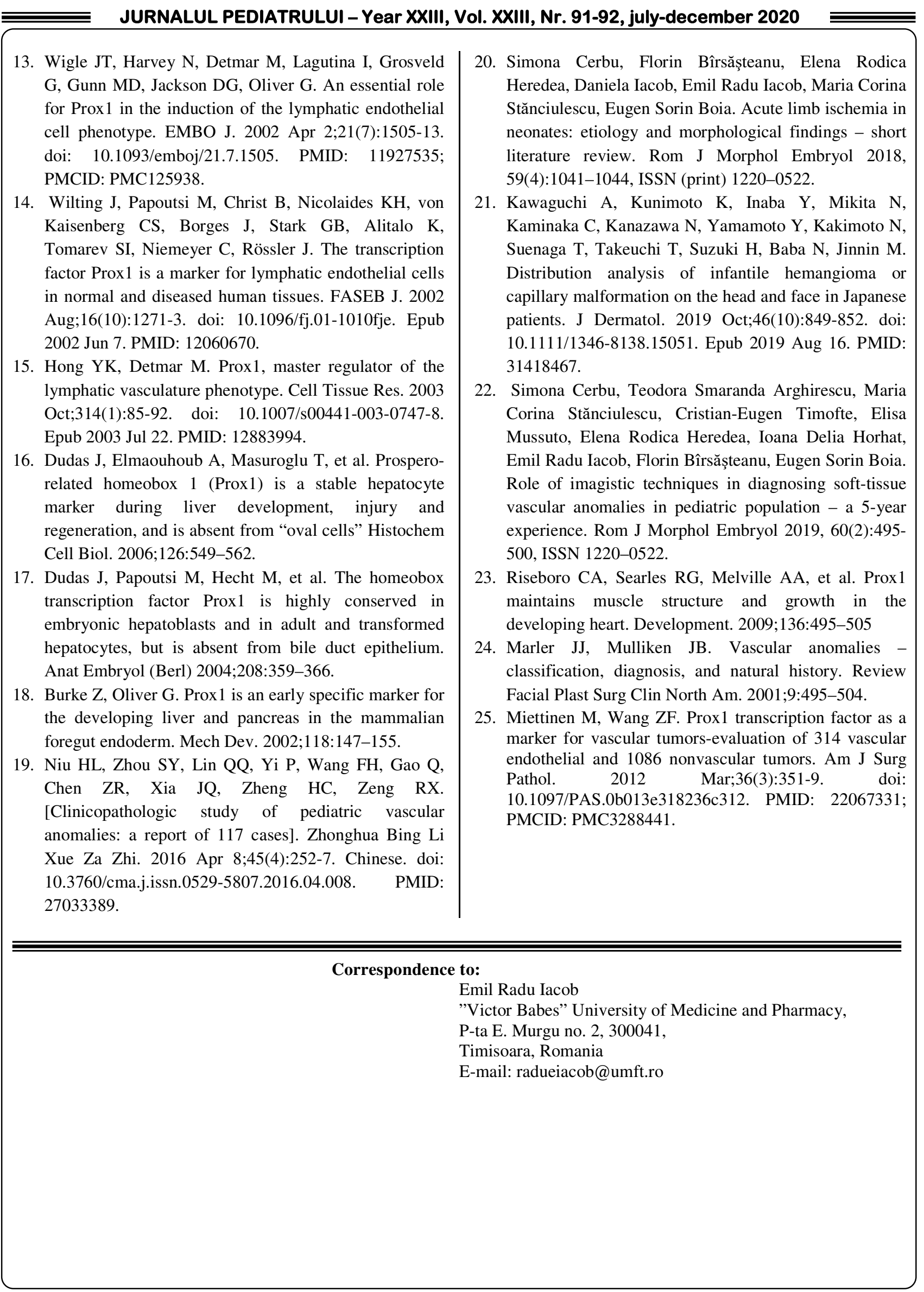

